\title{
Effets des prétraitements sur la germination des graines de Tamarindus indica L. (Fabaceae-Ceasalpinoideae) en pépinière : proposition pour une restauration de l'espèce au sahel.
}

Amadou GARBA ${ }^{1}$, Abdou AMANI 2, Saley KARIM ${ }^{3}$, Boubé MOROU 3 , Abdoul Kader Soumaila SINA', Ali MAHAMANE 4

1. Université Abdou Moumouni de Niamey, Faculté des Sciences et Techniques, Département de Biologie, Laboratoire GARBA Mounkaila, BP 1066, Niamey, Niger Tel : (+227)97569925 Tel +227)96296001 Email aksoumailasina@gmail.com

2. Institut National de la Recherche Agronomique du Niger (INRAN), BP 429 Niamey, Niger, Tel : (+227)96977476 Email : amaniabdou19@yahoo.fr

3. Université Dan Dicko Dankoulodo de Maradi, Niger. Tel (+227) 96572215 Email : boubem@yahoo.fr, Tel (+227)96103568, Email : karim saley@yahoo.fr,

4. Université de Diffa, Faculté des Sciences Agronomiques, BP 78, Diffa, Niger. Tel (+227)96967724 Email: alimahamane3@gmail.com

Auteur correspondant : Tel :(+227)97569925 Email : garbaamadou10@yahoo.fr

Original submitted in on $13^{\text {th }}$ March 2020. Published online at www.m.elewa.org/journals/ on $31^{\text {st }}$ May 2020 https://doi.org/10.35759/JABs.149.10

\section{RÉSUMÉ}

Objectif : L'aridité du climat sahélien et les pressions anthropiques sur la végétation ligneuse handicapent la régénération en milieu naturel des certaines espèces légumineuses d'importance socioéconomique, comme le tamarinier. La présente étude, conduite dans le jardin botanique de la Faculté des Sciences et Techniques de l'Université Abdou Moumouni de Niamey, a pour objectif d'évaluer l'effet des trois prétraitements sur la germination des graines de Tamarindus indica en pépinière.

Méthodologie et résultats: la méthodologie a consisté à soumettre les graines à trois prétraitements différents : (i) le graines trempées dans l'eau bouillie à $100^{\circ} \mathrm{C}$ jusqu' à refroidissement, (ii) le trempage des graines dans l'eau tiède pendant 72 heures et (iii) les graines scarifiées. Les graines non prétraitées ont été considérées comme témoins.

Conclusion et application des résultats : Sur 320 graines semées, 113 graines ont germé soit un taux de germination total de $35,31 \%$ et un taux de mortalité de $64,69 \%$. Le meilleur taux de germination a été obtenu avec les graines scarifiées $(\mathrm{T} 4=42,5 \%)$, suivi des graines trempées dans l'eau bouillante (T2 = $37,5 \%)$, puis les graines témoins $(\mathrm{T} 1=32,5 \%)$. Le plus faible taux de germination a été obtenu au niveau des graines trempées dans l'eau tiède $(T 3=28,75 \%)$. L'ensemble de ces résultats ont été assez satisfaisants. Les résultats de cette étude peuvent avoir des conséquences pratiques en termes de gestion des populations de $T$. indica. Ils montrent que la scarification et l'eau bouillante permettent d'obtenir des taux de germination des graines assez satisfaisants. Ces techniques peu coûteuses et accessibles à tous, peuvent être considérées comme des moyens pour produire facilement les plants de cette espèce et réhabiliter ainsi les zones favorables à sa conservation au Niger.

Mots clés : Germination, Tamarindus indica L., Légumineuses, Jardin botanique, Niamey, Niger 
Effects of pre-treatments on germination of Tamarindusindica L. seeds (Fabaceae-Ceasalpinoideae) in nurseries: proposal for a restoration of the species in the Sahel.

\begin{abstract}
Objective: The aridity of the Sahelian climate and anthropogenic pressures on woody vegetation hamper the regeneration in the natural environment of certain leguminous species of socio-economic importance, such as the tamarind tree. The present study, conducted in the Botanical Garden of the Faculty of Science and Technology of the Abdou Moumouni University of Niamey, aims at evaluating the effect of three pretreatments on the germination of Tamarindus indica L. seeds:

Methodology and results: the methodology consisted in subjecting the seeds to three different pretreatments. (i) Soaking the seeds in boiled water at $100^{\circ} \mathrm{C}$ until cooling, (ii) soaking the seeds in warm water for 72 hours and (iii) scarifying. Non-pre-treated seeds were considered as controls. Out of 320 sown seeds, 113 seeds germinated, giving a total germination rate of $35.31 \%$ and a mortality rate of $64.69 \%$. The best germination rate was obtained with scarified seeds (T4 $=42.5 \%)$, followed by seeds soaked in boiling water $(\mathrm{T} 2=37.5 \%)$, then the control seeds $(\mathrm{T} 1=32.5 \%)$. The lowest germination rate was obtained with seeds soaked in warm water $(\mathrm{T} 3=28.75 \%)$. All these results were quite satisfactory.

Conclusion and application of results: The results of this study may have practical consequences in terms of the management of Tamarindus indica L. populations. They show that scarification and boiling water can achieve satisfactory seed germination rates. These techniques, which are inexpensive and accessible to all, can be considered as means to easily produce plants of this species and thus rehabilitate areas favourable to its conservation in Niger.
\end{abstract}

Keywords: Germination, Tamarindus indica L., Legumes, Botanical garden, Niamey, Niger

\section{INTRODUCTION}

Au sahel, les essences fruitières forestières (EFF) locales jouent un rôle important dans la vie socioéconomique des ménages ruraux. Ces plantes tiennent une place importante dans les régimes alimentaires et atténuent les problèmes d'insécurité alimentaire (Getahun, 1990; Dan Guimbo et al., 2011).Cependant, depuis plus d'une décennie, les sécheresses répétitives et l'anthropisation croissante, ont provoqué une dégradation sans précédente des formations forestières dans les zones arides et semi-arides. C'est ainsi qu'aujourd'hui, la problématiques environnementales la plus préoccupante du 21 ème siècle juste après les changements climatiques constitue la déforestation (Word Bank, 2003). Selon les données les plus récentes (FAO, 2006, Doucet, 2007), les forêts couvraient environ 4 milliards d'hectares en 2005 , soit $30 \%$ des terres émergées. La perte nette de superficie forestière par année, pour la période 2000-2005, a été de 7,3 millions d'hectares. Outre son impact sur la biodiversité, cette déforestation, plus particulièrement en zone intertropicale, interviendrait pour plus de $20 \%$ des émissions annuelles de gaz à effet de serre (Doucet, 2007). Les effets conjugués de ces gaz à effet de serre, causes du réchauffement climatique, continuent de paralyser le domaine socioéconomique des pays du tiers monde. L'exploitation irrationnelle des forêts tropicales, réservoirs abritant plus de la moitié des espèces de la planète, se solde par une perte des ressources génétiques à grande vitesse (Millogo, 2014). Face à l'ampleur et à l'irréversibilité des menaces qui pèsent sur les forêts tropicales, la perte effective et potentielle des espèces couplées à celle des écosystèmes, constitueraient l'un des plus grands désastres environnementaux de notre ère (Kafando, 2016). $\mathrm{Au}$ sahel en général et au Niger en particulier, depuis les années des grandes sécheresses de 1972/1973, les effets conjugués de l'aridité du climat et de la croissance démographique ont conduit à une forte dégradation des systèmes écologiques, en occurrence les formations forestières, avec pour conséquence, la disparition des plusieurs espèces végétales et animales 
emblématiques. Les facteurs majeurs de dégradation de ces ressources forestières sont principalement imputés aux défrichements agricoles anarchiques, la coupe abusive du bois, le surpâturage, la mortalité naturelle des espèces due aux aléas climatiques. Au Niger, l'état de dégradation des ressources naturelles en particulier la végétation ligneuse des parcs agroforestiers, constitue une entrave majeure au développement socioéconomique du pays et au bien-être de sa population. Les ligneux jouent cependant un rôle essentiel dans la vie des populations sahéliennes en constituant non seulement un fourrage de relais des pâturages herbacés en saison sèche mais aussi une ressource fourragère stable pendant tout le cycle annuel et moins tributaire de la répartition des pluies de la saison précédente. De plus, ils apportent un complément alimentaire et sont utilisés comme bois de service, bois d'œuvre, médicaments (Lykke et al, 2004, Ganaba, 2005). En plus de leur utilisation dans la médecine traditionnelle, de nombreux extraits de plantes sont utilisés dans l'industrie pharmaceutique pour la fabrication de médicaments modernes et font l'objet d'un commerce international (Coppen 1995). Face à l'ampleur du défi à relever et pour protéger les ressources forestières ligneuses, le Ministère en charge de l'environnement et du développement durable a développé plusieurs techniques/technologies et stratégies de réhabilitation des écosystèmes dégradés, en vue de satisfaire les besoins des populations. Ces techniques ou technologies ont pour objectif de restaurer les terres dégradées, de reconstituer le couvert végétal, mais également d'améliorer les conditions de vie des populations en assurant la sécurité alimentaire. Cependant, les objectifs visés à travers ces programmes, n'ont pas pu parfois être atteints, à cause de l'absence de programmes scientifiques de plantation de nombreuses essences forestières indigènes d'importance socioéconomique, et cela pour plusieurs raisons parmi lesquelles : la faible disponibilité des graines liée aux problèmes de conservation, la pureté des graines, la dormance des graines, la croissance lente inhérente etc. Ces espèces forestières d'intérêt socioéconomique sont dans la plupart des cas contraintes à une surexploitation abusive par les populations et présentent des densités très faibles. A cet effet, en zone sahéliennes, peu de travaux ont été publiés sur les caractères adaptatifs des certaines espèces ligneuses alimentaires comme Faidherbia albida (Billand, 1990, Bastide, 1996, Diallo et al., 2008), Tamarindus indica (Diallo, 2001), Adansonia digitata (Assogbadjo, 2006) et Vitellaria paradoxa (Diarrassouba, 2008). De ces espèces, Tamarindus indica qui constitue notre matériel biologique, est une essence forestière dont, l'intérêt socioéconomique n'est plus à démontrer aujourd'hui. Des études récentes (Garba et al., 2017), ont montré que toutes ses parties sont sollicitées par les populations. Soumise à une exploitation abusive avec des peuplements vieux et épars, l'espèce présente des densités très faibles avec un taux de régénération nul (Douma et al 2010 ; Garba et al., 2017). En effet, les fruits de cette espèce qui sont très sollicités dans la fabrication de boisson traditionnelle, sont arrachés à l'état immature par les populations. D'autre part, les éleveurs coupent et émondent les branches et les feuilles pour fournir du fourrage aux animaux en saison sèche. En outre, l'effet de l'aridité du climat sahélien, constitue la seconde cause de la mortalité des individus de l'espèce en milieu naturel. Ces différentes pressions anthropiques limitent non seulement la production en fruits de l'espèce, mais aussi entrave sa régénération en milieu naturel. Pour mieux lutter contre la mortalité des individus de l'espèce, on doit chercher des solutions qui permettent de réhabiliter les peuplements dans les écosystèmes naturels, et résoudre le problème de sa régénération naturelle. D'où l'intérêt de cette étude dont l'objectif est d'évaluer l'effet des prétraitements sur la germination des graines du tamarinier (Tamarindus indica).

\section{MATÉRIEL ET MÉTHODES}


Site d'expérimentation; La présente étude d'essai d'expérimentation sur la germination des graines du tamarinier (Tamarindus indica), a été conduite en pépinière, dans le jardin botanique de la Faculté des Sciences et Techniques (FAST) de l'Université Abdou Moumouni de Niamey ( ${ }^{\circ} 13^{\circ} 30^{\prime} 09,4^{\prime \prime}$ E002 $\left.05^{\prime} 56,3^{\prime \prime}\right)$ (Figure 1a). Sa superficie est de 5,19 ha. Selon la carte de subdivision phytogéographique du Niger (Saadou, 1990), la zone se situe dans le climat sud-sahélien occidental B1 (figure1b). Cette zone se caractérise par une pluviométrie moyenne annuelle de $400 \mathrm{~mm}$. Le sol est de type sablo-limoneux. L'essai d'expérimentation a duré cinq (05) mois (août-décembre 2019. Avec une flore assez diversifiée, le jardin constitue à la fois un cadre de vie récréative pour les usagers, mais aussi un habitat écologique pour la petite faune. Cependant, la principale contrainte pour la survie des espèces végétales demeure l'absence d'un plan d'aménagement et de gestion durable.

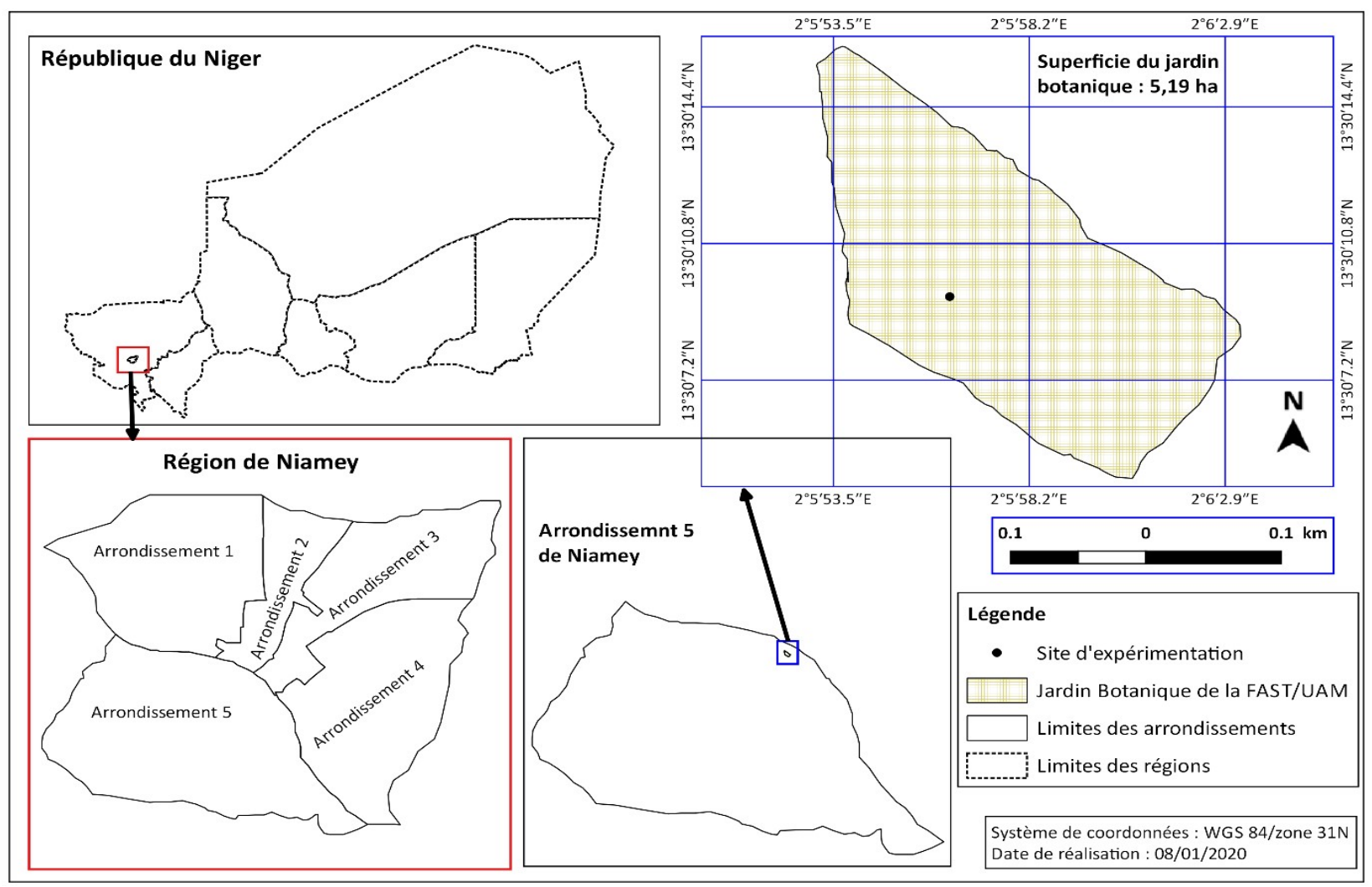

Figure 1a : Carte du jardin botanique 


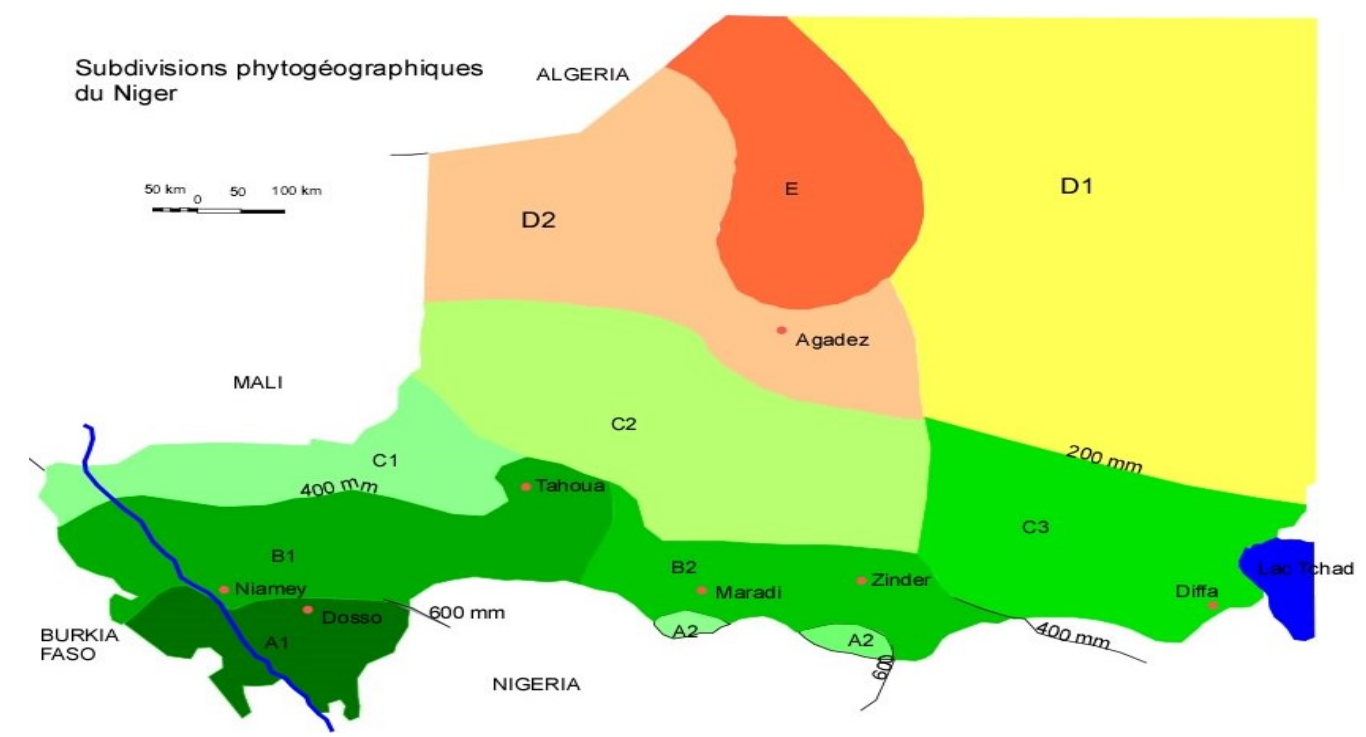

Figure 1b : Carte de subdivision phytogéographique du Niger (source : SAADOU, 1990,Karim, 2013)

A1: Compartiment Nord-soudanien occidental, A2 : Compartiment Nord-Soudanien central B1: Compartiment Sud-Sahélien occidental, B2 : Compartiment Sud-sahélien central Cl : Compartiment Nord-sahélien occidental, C2 : Compartiment Nordsahélien central C3 : Compartiment Nord-sahélien oriental, DI :Compartiment Sud-saharien central, D2 : Compartiment Sudsaharien oriental, $\mathrm{E}$ : Compartiment Sud-saharien montagnard

\section{Méthodes}

Collecte des semences: Des gousses mures et sèches ont été collectées entre octobre et décembre dans la commune rurale Torodi. La collecte a été faite au moyen des gaules d'Eucalyptus camaldulensis de 5 à $6 \mathrm{~m}$ de long et au bout desquelles sont fixés des crochés métalliques. Le choix des semenciers est basé sur des critères tels que : la distance entre les individus (1 à $2 \mathrm{~km}$ ), l'état sanitaire des individus et la production fruitière. L'ensemble des fruits collectés dans des sacs de 50 à $100 \mathrm{~kg}$ (figure 2), ont été mélangés et ensuite subdivisés en lots de fruits de tailles et de diamètres différents. Après échantillonnage, un seul lot des fruits a été retenu pour la germination. Ces fruits retenus n'ont pas été attaqués par les insectes bioravageurs. Après ce processus, les fruits ont été débarassés de leur coque, ensuite les graines à leur tour ont été séparées de leur pulpe. Les graines obtenues ont été subdivisées en deux lots : un lot de graines de diamètre et de taille petites et un lot des graines de diamètre et de taille moyens. Pour ce travail, c'est le lot des graines de diamètre et de taille moyens qui a été retenu (Figure 3).

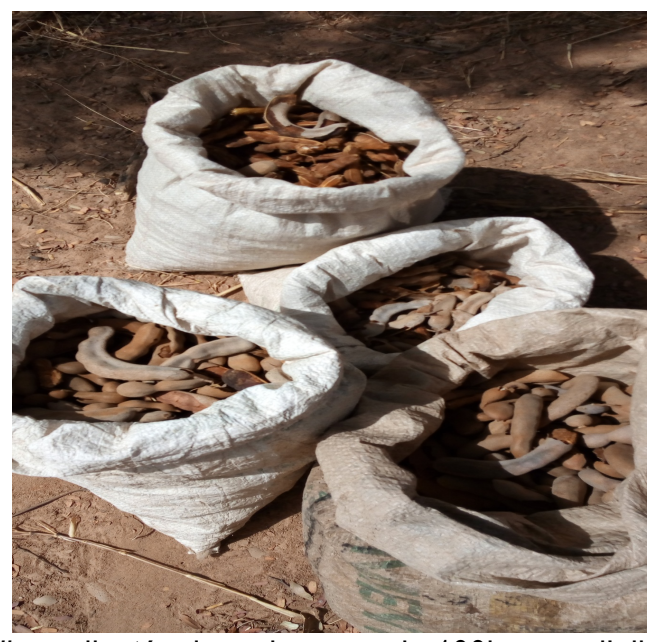

Figure 2 : Fruits de $T$. indica collectés dans des sacs de $100 \mathrm{~kg}$ pour l'alimentation humaine 
Garba et al., J. Appl. Biosci. 2020 Effets des prétraitements sur la germination des graines de Tamarindus indica L. (Fabaceae-Ceasalpinoideae) en pépinière : proposition pour une restauration de l'espèce au Sahel.

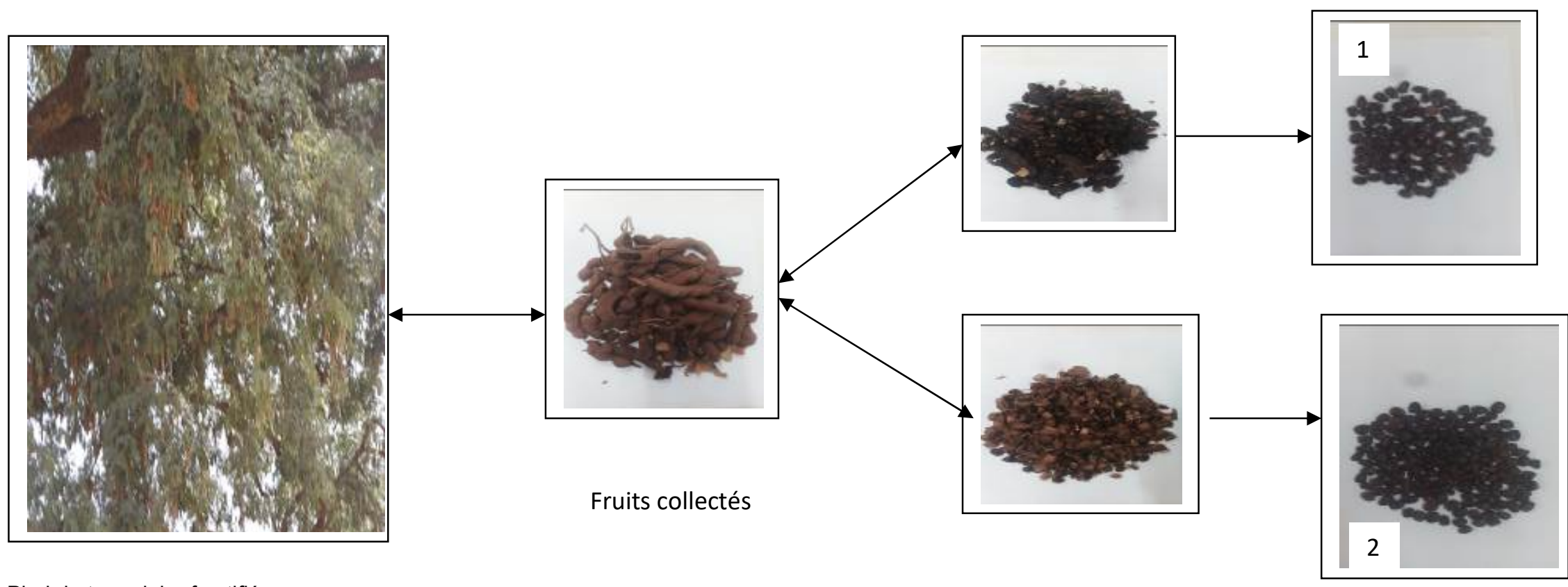

Pied de tamarinier fructifié

Fruits débarrassés

Débarrassés de leurs coques Lots des graines

sélectionnées

1. Lot des graines des diamètres et tailles moyennes

2. Lot des graines des diamètres et tailles petite

Figure 2 : Diagramme de la collecte des fruits et sélection des graines pour la germination 
Mise en place des semis : Pour vérifier la pureté des graines, ces dernières ont été déposées dans un seau contenant de l'eau du robinet. Sont considérées comme pures, les graines qui sont submergées et impures ceux qui sont restées à la surface de l'eau. Avant la mise en place des semis, les graines ont été subdivisées en quatre (04) lots de quatre-vingt (80) graines, soit au total 320 graines. Chaque lot de graines a subi un type de prétraitement. Ainsi, les graines du lot 1 constituent le témoin (T1), c'est-à-dire sans traitement quelconque, les graines du lot 2 (T2) ont été trempées dans de l'eau bouillie à $100^{\circ} \mathrm{C}$, jusqu'à refroidissement total, les graines du lot 3 (T3), ont été trempées dans de l'eau tiède pendant 72 heures, quant aux graines du lot 4 (T4), elles ont été scarifiées. La scarification consiste à pratiquer manuellement une incision superficielle et locale de la graine du pôle opposé à l'embryon à l'aide d'une lame afin d'enlever le tégument. Après traitement, chaque lot des graines est rincé à l'eau distillée, puis séché à l'air libre. Les différents traitements se présentent comme suit :

- $\quad T_{1}$ : graines témoins ;

- $\quad T_{2}$ graines trempées dans l'eau chaude ;

- $\mathrm{T}_{3}$ : graines trempées dans l'eau tiède pendant 72 heures :

- $\quad \mathrm{T}_{4}$ : graines scarifiées.

Les graines ont été semées dans des pots plastiques de 12 litres à une profondeur de $1,5 \mathrm{~cm}$. Chaque pot est rempli du substrat constitué de $2 / 3$ de sable pour $1 / 3$ de la matière organique. Le dispositif d'expérimentation est constitué de quatre (04) blocs ou répétions $(\mathrm{Ri})$. Chaque répétition est composée des vingt (20) pots, ce qui fait au total quatre-vingt (80) pots et dans chaque pot deux graines ont été semées. Le dispositif de germination est de type split plot avec quatre répétitions (Tableau 1).

Tableau 1: Dispositif d'expérimentation

\begin{tabular}{|c|c|c|c|c|c|c|c|}
\hline & & & REPETITIONS & & & & \\
\hline & R1 & & & & R2 & & \\
\hline P2T3 & P4T3 & P3T4 & P5T4 & P5T3 & P5T2 & P2T2 & P2T1 \\
\hline P2T1 & P1T2 & P1T4 & P1T3 & P4T2 & P2T3 & P5T1 & P1T3 \\
\hline P5T1 & P4T1 & P2T2 & P5T3 & P3T4 & P3T1 & P4T3 & P2T4 \\
\hline P3T1 & P1T1 & P5T2 & P3T2 & P1T4 & P4T4 & P3T3 & P4T1 \\
\hline P3T3 & P4T2 & P4T4 & P2T2 & P1T2 & P5T4 & P1T1 & P3T2 \\
\hline & R3 & & & & R4 & & \\
\hline P1T1 & P3T2 & P5T4 & P3T4 & P5T4 & P5T3 & P2T1 & $\mathrm{P} 1 \mathrm{~T} 2$ \\
\hline P4T1 & P3T3 & P1T2 & P1T4 & P3T1 & P1T4 & P1T1 & P3T2 \\
\hline P1T3 & P5T1 & P4T4 & P2T4 & P2T3 & P5T2 & P3T4 & P4T1 \\
\hline P4T3 & P4T2 & P2T3 & P5T3 & P2T2 & P4T2 & P2T4 & P4T4 \\
\hline P5T2 & P3T1 & P2T2 & P2T1 & P5T1 & P1T3 & P3T3 & P4T3 \\
\hline$P^{*}=$ pot & & & & & & & \\
\hline
\end{tabular}

L'essai d'expérimentation a duré cinq (05) mois (aoûtdécembre), soit 150 jours. Durant cette période, les semis ont été arrosés avec l'eau du robinet une fois par jour. Parfois l'arrosage peut aller jusqu'à trois jours après en cas des pluies. Une graine germe lorsque sa plantule est au-dessus du sol contenu dans le pot. Dès la germination, les paramètres relevés chaque jour sont

- le nombre de graines germées dans chaque répétition et pour chaque type de traitement, ceci pour calculer le pourcentage de germination (PG) dont la formule est la suivante :

$$
P G=\frac{\text { total des graines germées }}{\text { total des graines gemées }} \times 100 \quad \text { la }
$$

période de dormance (PD) qui est le nombre de jour entre le semis et le début de germination

- le nombre maximal de graines germées (NMG) pour chaque type de traitement.

- la capacité de germination qui est le nombre maximal des graines germées ;

- le nombre total des graines germées au dernier jour ;

- $\quad$ la teneur en eau (Te) par rapport au poids frais et au poids sec de la biomasse a été ensuite calculée respectivement par les formules suivantes utilisées par Ahoton (2009) dans la détermination de la teneur en 
eau des graines de Prosopis africana (Guill., Perrot. et Rich.) Taub.

$\mathrm{Te}=100 \times(\mathrm{pf}-\mathrm{ps}) / \mathrm{pf}$ et Te $=100 \times(\mathrm{pf}-\mathrm{ps}) / \mathrm{ps}$

Suivi de la croissance et variables mésurées des plants : Sur les plantules âgées de trois mois, nous

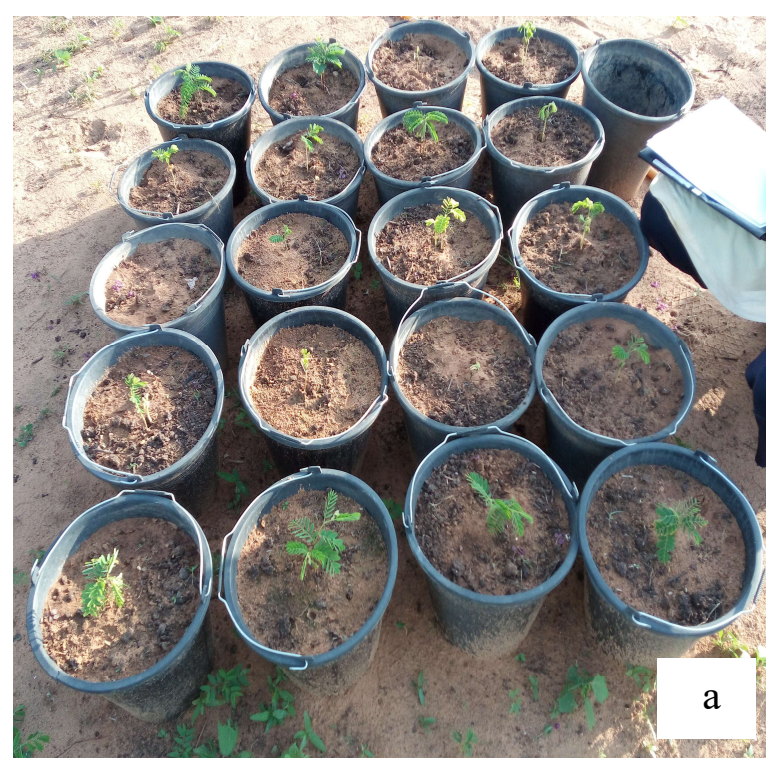

Figure 3 : Suivi de la germination et de la croissance des a) Relevés de nombre graines germées/jours

\section{Evaluation de la biomasse des tiges et des racines :} Un mois après le suivi de la germination des plantules, une répétition (R4), a été sacrifiée pour évaluer la contribution en biomasse de la tige et la racine des plantules. Pour évaluer cette biomasse, les plantules ont été déterrées des pots (figure 7 a) et classées par type de prétraitement. Au sein de chaque prétraitement, les plantules ont été coupées et divisées en deux parties : la partie aérienne (tige) et la partie souterraine (racine). Pour chaque partie, la biomasse fraiche a été pesée à l'aide d'un peson électrique. Les échantillons avons relevé la hauteur, le diamètre au collet de la tige principale, la hauteur de la première ramification, la longueur de la racine principale. Le rapport (hauteur de la tige /longueur de la tige principale). La contribution en biomasse des parties aérienne et souterraine a été aussi relevée (figure 3).

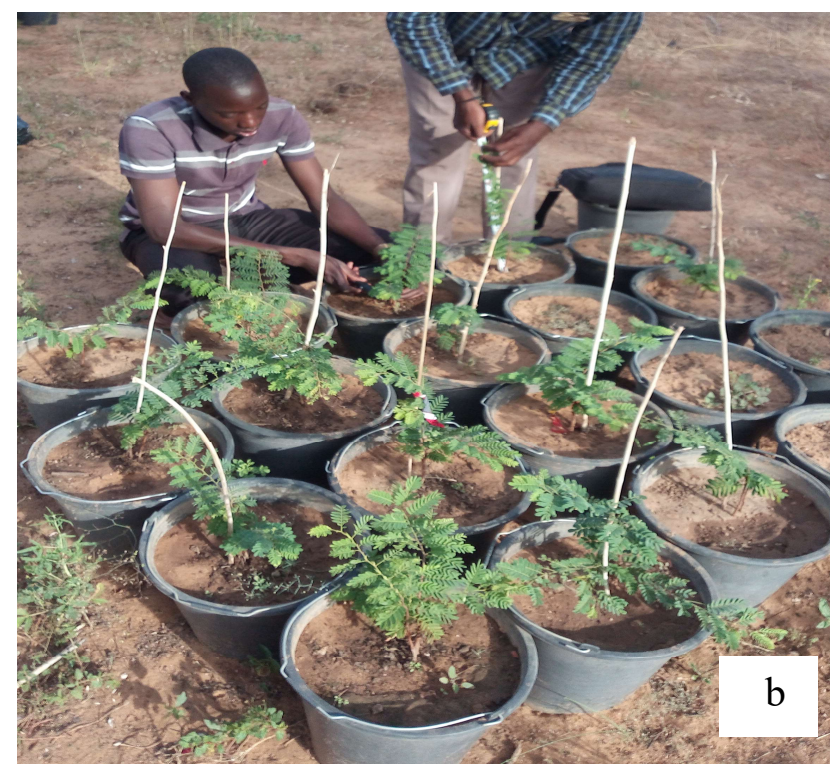

graines en pépinière

b) Mesure des hauteurs et des diamètres

des biomasses fraiches ont été par la suite séchés au soleil pendant une semaine puis repesées.

Traitements et analyse des données: Le tableur Excel 2007 a été utilisé pour la saisie des valeurs des différentes variables mesurées ainsi que l'élaboration des courbes de germination. Les analyses statistiques des variances ont été effectuées par le logiciel Minâtab 14. Le test statistique (ANOVA one-Way) a permi de comparer les différentes moyennes. L'analyse en composante principale(ACP) a été faite par le logiciel PCORD 5. 


\section{RÉSULTATS}

Composition floristique du jardin botanique: Un inventaire floristique non exhaustif a permi de recenser vingt-cinq (25) espèces ligneuses, réparties en 18 familles (Tableau 2), dont les plus importantes sont les Fabaceae-Mimosoideae (16\%), suivie des combretaceae (12\%).

Tableau 2 : Liste des espèces et des familles du jardin botanique

\begin{tabular}{|l|l|}
\hline Espèces & Familles \\
\hline Acacia nilotica (L.) Willd. ex Del. subsp. nilotica & Fabaceae-Mimosoideae \\
\hline Adansonia digitata L. & Bombacaceae \\
\hline Anogeissus leiocarpus (DC.) Guill. et Perr. & Combretaceae \\
\hline Azadirachta indica A. Juss. & Meliaceae \\
\hline Balanites aegyptiaca (L.) Del. & Balanitaceae \\
\hline Bauhinia rufescens Lam. & Fabaceae-Ceasalpinoideae \\
\hline Calotropis procera (Ait.) R. Br. & Asclepiadaceae \\
\hline Cassia sieberiana DC. & Fabaceae-Caesalpinioideae \\
\hline Cirtus aurantifolia & Rutaceae \\
\hline Detarium microcarpum & Fabaceae-Caesalpinioideae \\
\hline Diospyros mespiliformis Hochst. Ex A. DC. & Ebenaceae \\
\hline Eucalyptus camaldulensis Dehnh. & Myrtaceae \\
\hline Faidherbia albida (Del.) Chev. & Fabaceae-Mimosoideae \\
\hline Guiera senegalensis J.F. Gmel. & Combretaceae \\
\hline Jatropha curcas L. & Euphorbiaceae \\
\hline Lawsonia inermis L. & Lythraceae \\
\hline Maerua crassifolia Forsk. & Capparaceae \\
\hline Mangifera indica L. & Anacardiaceae \\
\hline Moringa oleifera & Moringaceae \\
\hline Parkia biglobosa (Jacq.) R.Br. ex Benth. & Fabaceae-Mimosoideae \\
\hline Prosopis africana (Guill. \& Perr.) Taub. & Fabaceae-Mimosoideae \\
\hline Tamarindus indica L. & Fabaceae-Ceasalpinoideae \\
\hline Terminalia mentaly H. & Combretaceae \\
\hline Vitex doniana Sweet. & Verbenaceae \\
\hline Zyziphus mauritania Willd & Rhamnaceae \\
\hline &
\end{tabular}

Effets des prétraitements sur la croissance des graines du tamarinier » Après la levée de dormance les graines de différents prétraitements ont commencé à germer. La période de germination (PG) ou temps de germination, le plus court a été observé au niveau du lot des graines scarifiées ( $P G=8$ jours) et celui du lot des graines trempées dans l'eau tiède ( $P G=8$ jours). Le neuvième jour le lot des graines témoins (T1) et celui des graines trempées dans l'eau chaude ont commencé à germer. Leur période de germination est la même et est de neuf (09) jours. Le plus grand taux de germination a été observé au niveau du lot des graines scarifiées (T4 $=42,5 \%$ ), suivi du lot des graines trempées dans l'eau bouillante ( $\mathrm{T} 2=37,5 \%$ ), puis au niveau du lot des graines témoins $(\mathrm{T} 1=32,5 \%)$. Le plus faible taux de germination a été obtenu au niveau $\mathrm{du}$ lot des graines trempées dans l'eau tiède ( $\mathrm{T} 3=$
$28,75 \%$ ). Le nombre maximal des graines germées par jour demeure respectivement douze (12) chez les graines scarifiées $(T 4=12)$, six $(06)$ chez les graines témoins $(T 1=6)$, cinq $(05)$ chez les graines trempées dans l'eau chaude ( T2 $=5$ ) et quatre $(04)$ chez les graines trempées dans l'eau tiède $(\mathrm{T} 3=4)$. La capacité de germination ou le nombre maximal des graines germées dans cette étude est 113. Le nombre de graines germées le dernier jour est un (01). Elle est du lot des graines trempées dans l'eau bouillie à $100^{\circ} \mathrm{C}$. Le test statistique de Ficher indique que la variation du cumul du nombre des graines germées par jour entres les différents prétraitements n'est pas significative $(P=$ 0,140 ) au seuil de $5 \%$. Le cumul du nombre des graines germées par jour et par traitement a permi de générer la courbe du taux de germination des graines du tamarinier (Figure 4). 


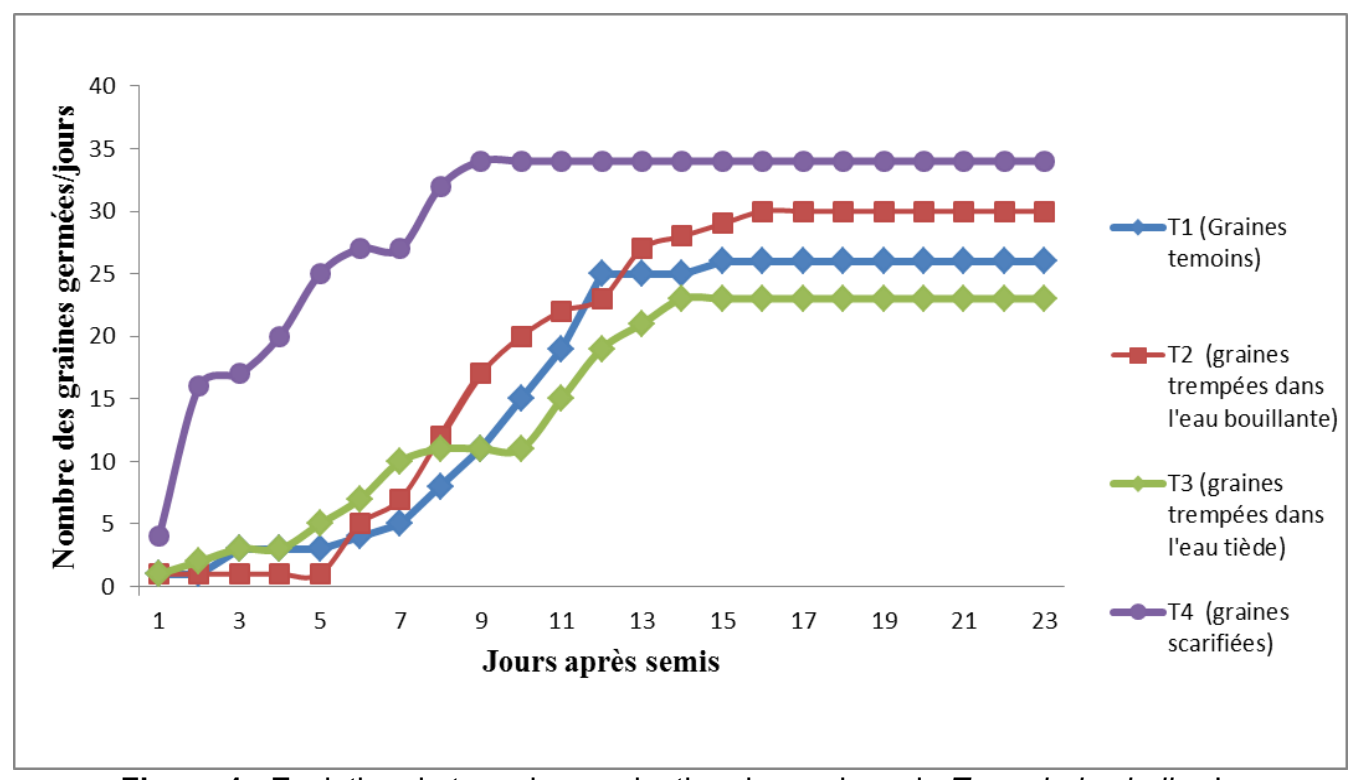

Figure 4 : Evolution du taux de germination des graines de Tamarindus indica L.

Taux de mortalité des graines: Un mois après le suivi de la germination, le taux de mortalité au sein des lots des graines a été évalué. Les résultats montrent que le plus fort taux de mortalité a été enregistré dans le lot des graines séjournées dans l'eau tiède $(\mathrm{T} 3=$ $71,25 \%)$, suivi du lot des graines témoins ( $\mathrm{T} 1=67,5 \%)$. Le plus faible taux de mortalité a été obtenu dans le lot des graines scarifiées ( $\mathrm{T} 4=57,5 \%)$, suivi du lot des graines trempées dans l'eau bouillante $(\mathrm{T} 2=62,5 \%)$.

Variation du diamètre au collet et de la hauteur des plantules du tamarinier: Le tableau 3 ci-dessous illustre les résultats sur la variation de la hauteur moyenne et du diamètre au collet moyen des plants du tamarinier selon les différents prétraitements. En ce qui concerne la hauteur moyenne, elle est respectivement de 35,8 $\pm 6,002 \mathrm{~cm}$ pour le lot des graines témoins ( $\mathrm{T} 1$ $=35,8 \pm 6,002 \mathrm{~cm}), 32,267 \pm 4,543 \mathrm{~cm}$ pour le lot des graines trempées dans l'eau bouillante (T2
$=32,267 \pm 4,543 \mathrm{~cm}), 31 \pm 8,075$ pour le lot des graines séjournées dans l'eau tiède $(\mathrm{T} 3=31 \pm 8,075 \mathrm{~cm})$ et $31,635 \pm 8,59 \mathrm{~cm}$ pour les graines scarifiées ( $\mathrm{T} 4=$ $31,635 \pm 8,59 \mathrm{~cm})$. Le test de Ficher montre que la variation des hauteurs des différents des plants issus des quatre types des traitements n'est pas significative $\left(P^{*}=0,226\right)$ au seuil de $5 \%$. Pour ce qui est du diamètre moyen au collet, il est de 5,967 $\pm 0,965 \mathrm{~cm}$ pour le lot des graines témoins $(T 1=5,967 \pm 0,965)$ $\mathrm{cm}, 5,759 \pm 1,168 \mathrm{~cm}$, pour le lot des graines trempées dans l'eau chaude (T2 =5,759 $\pm 1,168 \mathrm{~cm}$,), $5,434 \pm$ $1,01 \mathrm{~cm}$, pour le lot des graines séjournées dans l'eau tiède $(\mathrm{T} 3=5,434 \pm 1,01 \mathrm{~cm}$,) et 6,195 $\pm 1,572 \mathrm{~cm}$, pour les graines scarifiées ( $\mathrm{T} 4=6,195 \pm 1,572 \mathrm{~cm}$,). Le test d'analyse de Ficher montre la variation du diamètre au collet des plants n'est significative $(P=0,440)$ au seuil de $5 \%$.

Tableau 3 : Hauteur et diamètre au collet moyens des plantules du tamarinier

\begin{tabular}{|c|l|c|c|}
\hline & Hauteurs moyennes $(\mathbf{C m})$ & Diamètre moyen $(\mathbf{C m})$ & CV$^{*}$ \\
\hline T1 & $35,8 \pm 6,002$ & $5,967 \pm 0,965$ & 16,17 \\
\hline T2 & $32,267 \pm 4,543$ & $5,759 \pm 1,168$ & 20,28 \\
\hline T3 & $31 \pm 8,075$ & $5,434 \pm 1,01$ & 18,58 \\
\hline T4 & $31,635 \pm 8,59$ & $6,195 \pm 1,572$ & 25,38 \\
\hline & $\mathrm{P}^{*}=0,226$ & $\mathrm{P}^{*}=0,440 \mathrm{P}^{*}=$ probabilité & \\
\hline
\end{tabular}

Croissance en hauteur de la tige et des racines des plantules du tamarinier: Les résultats de la figure 5 , illustrent la croissance en hauteur de la tige et du pivot racinaire des plantules du tamarinier. On remarque qu'un mois après la germination des graines, la hauteur de la plantule a atteint $24 \mathrm{~cm}$, tandis que celle de la racine est de $3 \mathrm{~cm}$, soit une différence de $21 \mathrm{~cm}$ de plus pour la tige. Au deuxième et au troisième mois on 
constate que la tige et la racine des plantules croient au même rythme. Après le cinquième mois, on constate que la racine des plantules croit plus vite que celle de la tige, jusqu'au douzième mois où la longueur de la racine atteigne $61 \mathrm{~cm}$, tandis que celle de la racine est de $38 \mathrm{~cm}$ soit une différence de $23 \mathrm{~cm}$ de plus pour la racine. Cette croissance moyenne est respectivement $52,88 \pm 26,58 \mathrm{~cm}$ pour la racine et $33,94 \pm 7,29 \mathrm{~cm}$ pour la tige.

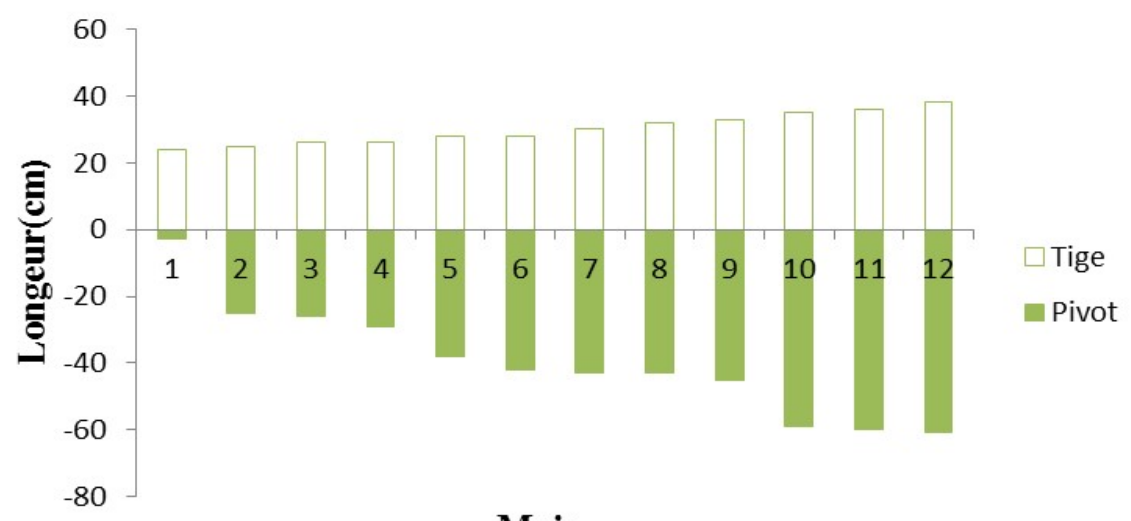

Mois

Figure 5 : Corrélation entre la croissance en hauteur de la tige et la racine des plantules

La courbe de régression (Figure 6) qui explique la relation entre la hauteur et le diamètre des plantules donne un coefficient de détermination $R^{2}=0,86$. Ce qui signifie qu'il a une bonne corrélation entre la hauteur et le diamètre au collet des plantules du tamarinier. Le résultat du test d'adéquation du modèle montre que la courbe de l'équation est effectivement linéaire $(P$ $<0,001$ ) au seuil $5 \%$.

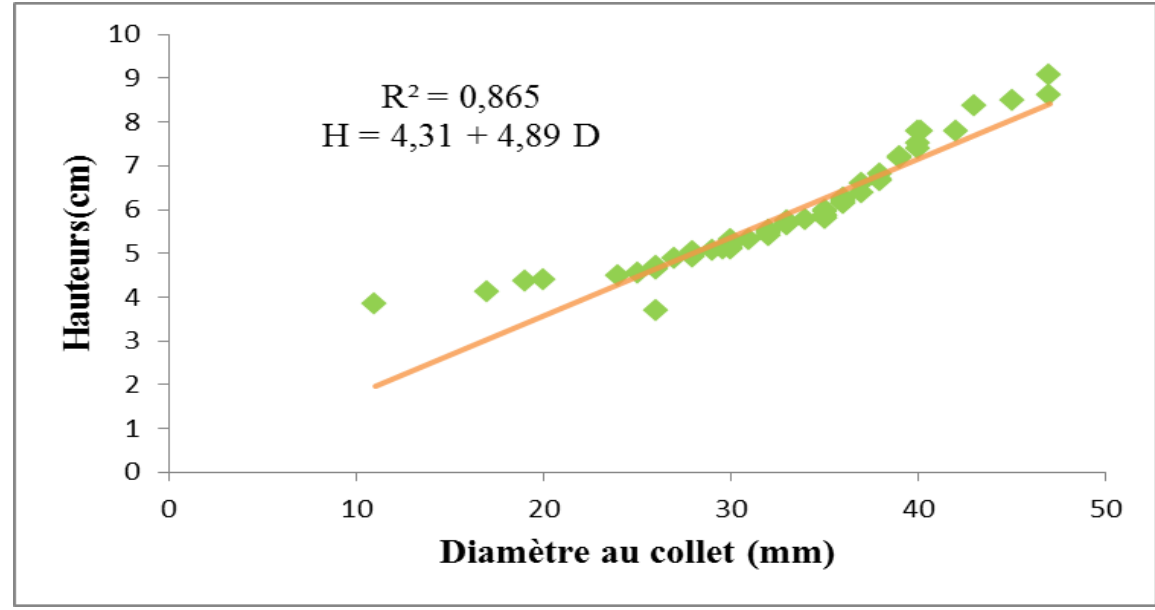

Figure 6 : Courbe de régression entre la hauteur et le diamètre au collet

Contribution en biomasse des tiges et des racines des plantules du tamarinier : Les résultats du tableau 4 illustrent la contribution en biomasse des tiges et des racines. La somme des biomasses sèche des différentes parties (tiges et racines) des plantules a permis de générer l'histogramme de biomasse ( la figure $7 \mathrm{~b}$. La teneur en eau de la tige et celle de la racine a été également déterminée (tableau 4). Ces résultats montrent qu'en terme de biomasse la partie aérienne (tige) contribue plus que la partie souterraine, soit respectivement $\left(B_{\text {tige }}=0,23 \mathrm{~kg}\right.$ et $\left.B_{\text {racine }}=0,195 \mathrm{~kg}\right)$. Le test statistique de Tukey's indique que la variation de la quantité de biomasse entre la tige et la racine n'est pas significative $(p=0,717)$ au seuil de $5 \%$. 
Tableau 4 : Biomasse des tiges et des racines

\begin{tabular}{|l|l|l|c|l|c|c|}
\hline & $\begin{array}{l}\text { Poids frais } \\
(\mathbf{k g})\end{array}$ & $\begin{array}{l}\text { Poids sec } \\
(\mathbf{k g})\end{array}$ & & \multicolumn{2}{l|}{$\begin{array}{l}\text { Poids } \\
\text { frais (kg) }\end{array}$} & $\begin{array}{l}\text { Poids } \\
\text { Sec (kg) }\end{array}$ \\
\hline Prétraitements & Tige & Tige & Teneur en eau & Racines & Racine & Teneur en eau \\
\hline T1 & 0,105 & 0,045 & 57,14 & 0,075 & 0,04 & 46,67 \\
\hline T2 & 0,04 & 0,025 & 37,5 & 0,06 & 0,04 & 33,33 \\
\hline T3 & 0,07 & 0,04 & 42,8 & 0,065 & 0,04 & 38,46 \\
\hline T4 & 0,135 & 0,12 & 11,11 & 0,11 & 0,075 & 31,82 \\
\hline Total & $\mathbf{0 , 3 5}$ & $\mathbf{0 , 2 3}$ & $\mathbf{1 4 8 , 6 1}$ & $\mathbf{0 , 3 1}$ & $\mathbf{0 , 1 9 5}$ & $\mathbf{1 5 0 , 2 8}$ \\
\hline
\end{tabular}
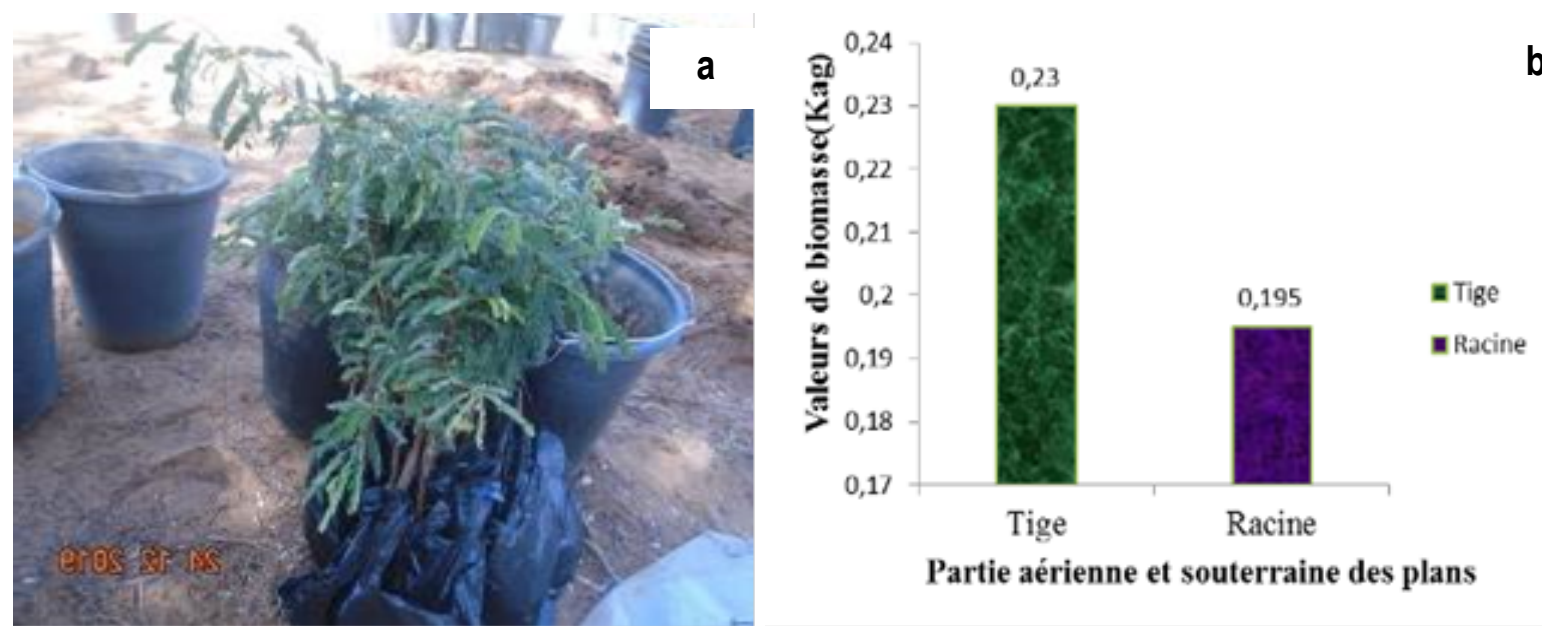

Partie aérienne et souterraine des plans

Figure 7 : Evaluation de la productivité primaire et de la teneur en eau des organes des plantules du tamarinier âgées de trois mois en pépinière a) plantules déterrées des pots b) Courbe de biomasse des tiges et des racines

Analyse multivariée: Les moyennes des longueurs des tiges et des racines des plants issus de quatre prétraitements ont été soumises à une analyse en composante principale (ACP). Les résultats donnent une inertie de $100 \%$ qui se répartit entre les deux axes. L'axe 1 explique à $90,168 \%$ la variabilité de la croissance en longueur des tiges. L'axe 2, quant à lui explique à $9,832 \%$ la croissance en longueur des racines (Figure 8). L'axe 1 corrélé à la longueur des tiges pourrait être considéré comme étant l'axe de la biomasse aérienne et l'axe 2 influencé par la longueur des racines, la biomasse souterraine ou racinaire. La matrice de corrélation des quatre prétraitements, soumise à une classification hiérarchique selon la méthode de Ward's a permis d'obtenir le dendrogramme (Figure 9). Au seuil de $79 \%$, on obtient trois groupements (G1, G2 et $G 3$ ). Le groupe $\mathrm{G} 1$ regroupe les prétraitements $T 1$ et $T 4$, le groupe $G 2$, le prétraitement T2 et le groupe G3 est constitué du prétraitement $\mathrm{T} 3$. 


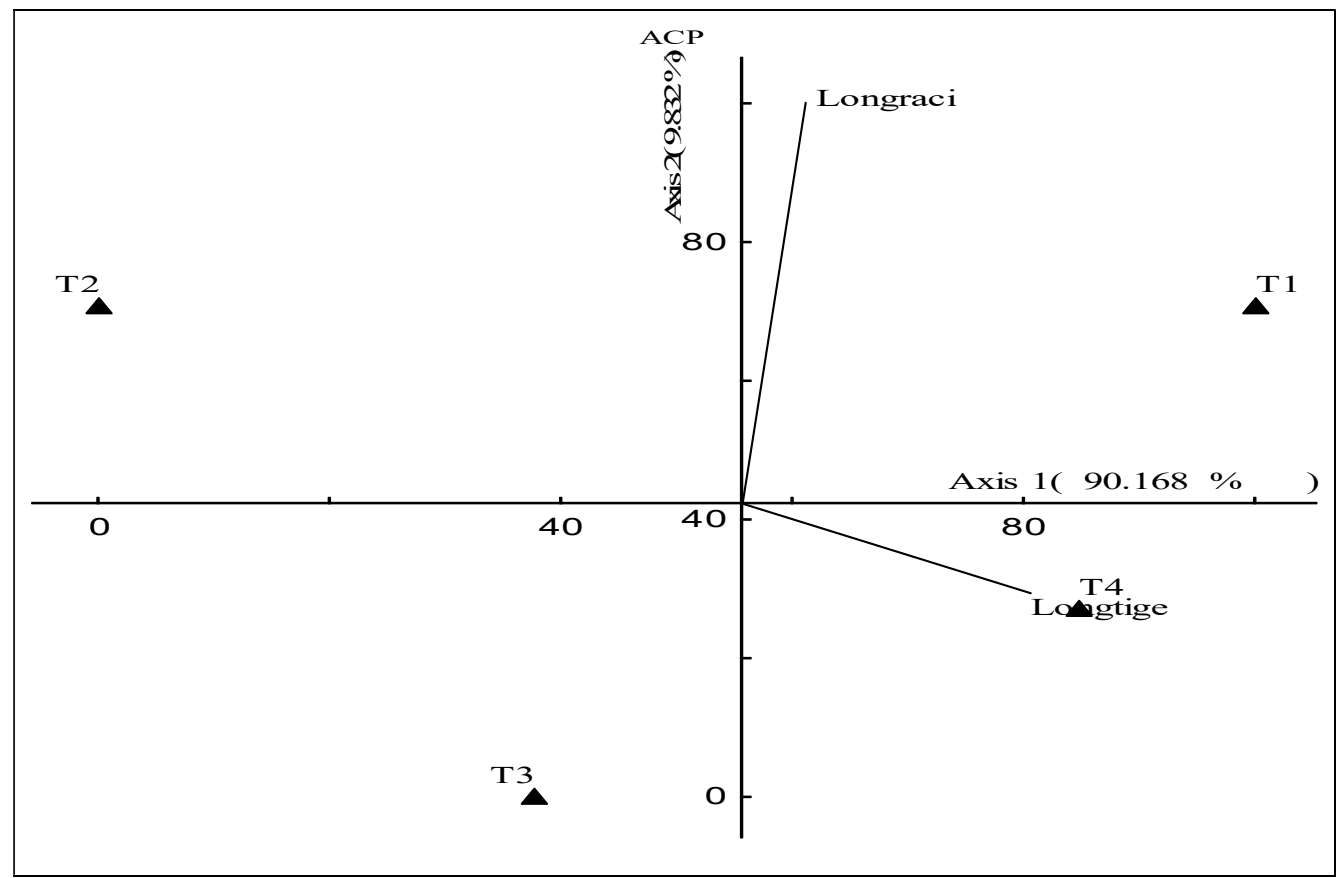

Longtige $=$ longueur des tiges $;$ Lonngraci $=$ longueur des racines

Figure 8 : Analyse en composente principale de la longueur des racines et des tiges

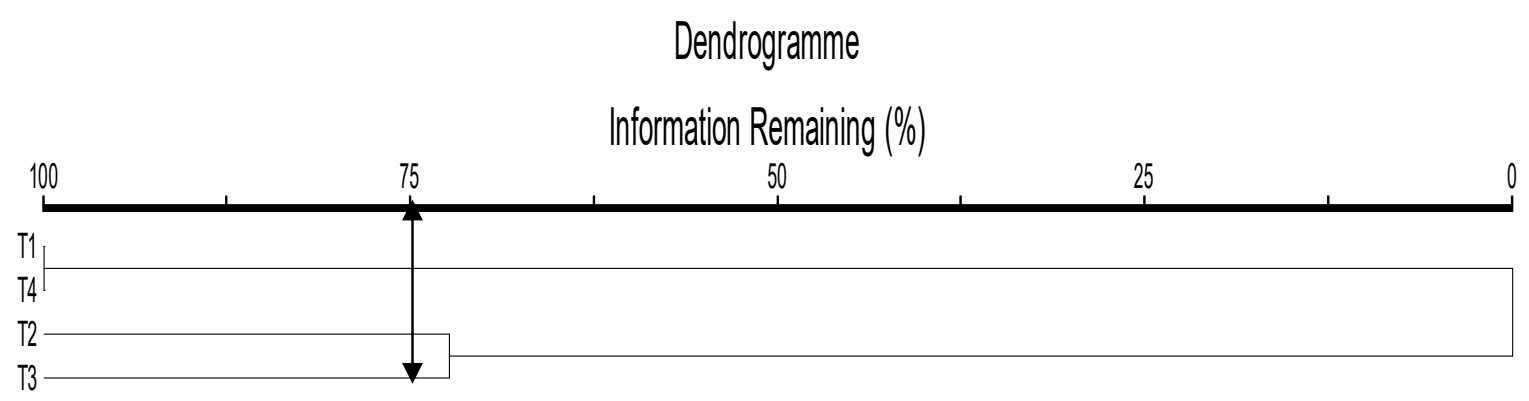

Figure 9 : Classification hiérarchique des trois prétraitements

\section{DISCUSSION}

Les graines de légumineuses des zones sèches sont généralement dures. Elles possèdent des téguments imperméables à l'eau et l'oxygène induisant une inhibition tégumentaire (Werker, 1981). Cette dormance permet la conservation à long terme des semences, mais nécessite d'être levée avant semis sous peine d'obtenir des germinations très erratiques (Cavanagh, 1987). A cet effet, la présente étude a permis de démontrer que pour avoir une germination rapide des graines de $T$.indica, le prétraitement des semences est nécessaire avant et après conservation de celles-ci. Le prétraitement raccourcit non seulement le délai de germination, mais aussi augmente le taux de germination. Les résultats de cette étude montrent que le délai et le taux de germination des graines varient suivant le type de prétraitement. C'est ainsi que pour les quatre lots des graines de $T$. indica prétraitées, le délai de germination maximum était de neuf (09) jours et le minimum huit jours (08). Le plus fort taux de germination a été observé chez les lots des graines scarifiées. II est suivi de celui des graines trempées dans l'eau bouillante à $100^{\circ} \mathrm{C}$. La scarification mécanique des graines accélère la germination, mais elle expose parfois l'embryon et le cotylédon gorgé d'eau et des protéines à des pourritures et aux attaques parasitaires (Ahoton et al., 2009). Ceci peut être la cause du taux de mortalité des graines observées dans lot. Le traitement à l'eau bouillante constitue également un moyen efficace pour accélérer la germination des graines, car celui-ci permet de ramollir les téguments de la graine et réduit l'imperméabilité de celui-ci à l'eau (Tran, 1984). Le taux de mortalité des graines 
observées dans ce lot pourrait être lié à la sensibilité de l'embryon à la chaleur en fonction de la durée du trempage. Ce qui indique que le temps de séjour des semences trempées dans l'eau chaud à $100^{\circ} \mathrm{C}$ jusqu'à refroidissement, serait trop long et aurait entrainé certainement la perte du pouvoir germinatif de certaines graines. Ces résultats sur l'impact de la chaleur sur le pouvoir germinatif des graines de $T$. indica, apportent des informations sur l'impact des températures extrêmes au sahel sur le pouvoir germinatif des graines de cette espèce en milieu naturel. Des résultats similaires à ceux de la scarification ont été obtenus par El hamdouni et al., (2001) sur la germination in vitro du fraisier (fragaria $x$ ananassa duch.) cvs 'chandler' et 'tudla'., Todd-bockarie et al., (1993) et Assongba et al., (2013) sur la germination de Dialium guineense Willd. Ces prétraitements ont permis également d'obtenir des résultats satisfaisants sur les semences d'autres légumineuses comme Parkia biglobosa Benth. et Prosopis africana (Guill., Perrot. et Rich.) Taub (FAO, 1992 ; Ahoton et al., 2009, Abubakar, 2013). Le lot des graines trempées dans l'eau tiède présente le plus faible taux de germination et le plus grand taux de mortalité. Ces résultats s'expliquent par l'impact de la durée du trempage qui pourrait toutefois affecter le pouvoir germinatif des certaines graines dont la vitesse de l'imperméabilité tégumentaire varie suivant l'état physiologique interne et externe de l'embryon. En effet, plus une graine est dure, plus elle résiste à l'effet des facteurs de levée de la dormance, plus la graine est tendre, plus elle résiste moins aux facteurs des conditions environnementales qui conditionnent sa germination. Les résultats du lot des graines témoins ont contribué à augmenter le taux de germination des graines. Le taux de mortalité enregistré peut être lié à la pureté des graines mais aussi aux conditions d'expérimentations. Des études récentes ont montré que les fruits de cette espèce sont attaqués par des insectes pendant le processus de la maturité. Ceux-ci pondent leurs œufs dans l'amande des fruits qui restent pendant plusieurs temps. Quand les œufs éclosent, les larves se nourrissent de l'amande des graines qui contient des composées glucidiques (Garba et al., 2019). Ces contraintes entomologiques diminuent la longévité des semences et la survie des plantules qui sont des facteurs déterminants pour la régénération séminale des espèces forestières en milieu naturel (Bariteau, 1992; Bationoa et al., 2000). Dans cette étude, les résultats sur le suivi de la croissance des plantules ont révélé que la racine des plants croit plus vite que la tige. La hauteur maximale des plantules de trois mois a été de $47 \mathrm{~cm}$, tandis que celle de la racine principale est $140 \mathrm{~cm}$. Ces plantules peuvent résister longtemps en cas de situation de déficit hydrique. La viabilité des semences et la résistance des plantules aux multiples agressions en milieu naturel peuvent être déterminées par des facteurs biologiques et écophysiologiques propres aux espèces elles-mêmes (Lopez et al., 2000). C'est le cas par exemple de la teneur en eau des graines qui affecte la longévité et les conditions de conservation des semences (OrozcoSegovia, 1993) De même, la morphologie fonctionnelle des plantules conditionne de façon significative l'adaptabilité de celles-ci aux facteurs souvent très contraignants du milieu naturel (Vetaas, 1992). Cette caractéristique écophysiologique et biologique du système racinaire, en occurrence la racine principale, assure aux individus de l'espèce, une capacité d'adaptation et de résistance aux aléas climatiques en zone sahélienne. Des résultats similaires au développement du système racinaire chez les plans de $T$. indica ont été observés chez les plants de Sclerocarya birrea (A. Rich.) Hochst (Alexandre, 1993 ; Pierre, 2003 ; Bellefontaine, 2005, Abdourhamane et al., 2014). Ces auteurs affirment que cette caractéristique physiologique pourrait être perçue en zone sahélo-soudanienne comme un critère favorable au processus d'adaptation des espèces ligneuses dans un environnement aride. Selon Dianda et al., (2010), un système racinaire vigoureux permettrait d'assurer une alimentation convenable en eau et en nutriments à partir des horizons profonds, et une bonne vigueur de reprise après le feu. Les trois prétraitements (eau bouillante, eau tiède, scarification) ont donné des résultats assez satisfaisants et la scarification semble le meilleur prétraitement pour obtenir un taux satisfaisant de germination des graines de cette espèce. Ces connaissances sont essentielles pour s'orienter progressivement vers la création des centres de collecte des semences et des programmes de sylviculture pour la production massive des plants des essences forestières en voie de disparition au sahel. Face à l'aridité du climat et aux mutilations anthropiques de plus en plus croissants sur les individus du $T$. indica, facteurs qui compromettent sa fructification et ses capacités à disséminer ses semences en milieu naturel, l'identification et la cartographie des aires favorables à la conservation de l'espèce s'avère indispensable pour une gestion plus rationnelle et durable au Niger. 


\section{CONCLUSION}

La régénération naturelle de $T$. indica se pose avec acuité dans les écosystèmes agroforestiers du sahel. A travers cette étude, l'application des prétraitements sur les graines a permis d'obtenir des meilleurs résultats sur le taux de germination. Ainsi, pour obtenir un taux de germination satisfaisant des graines de $T$. indica, la scarification mécanique constitue le meilleur choix, suivi de l'eau bouillante à $100^{\circ} \mathrm{C}$. Ces résultats constitueront sans doute des informations capitales pour les structures des formations forestières en particulier le centre national des semences forestières (CNSF) du

\section{REMERCIEMENTS}

Au sortir de cette étude, nous remercions tous les personnels du jardin botanique de la faculté des sciences et techniques en particulier les responsables du jardin botanique. Nous tenons à remercier vivement Mr. Harouna Moussa Bilali qui, malgré ses normes
Niger, car il s'agit des acquis scientifiques qui, nous l'espérons, vont susciter un plus grand intérêt débouchant sur de multiples études en ce qui concerne la restauration des essences forestières d'importance socioéconomique en voie de disparition au Niger. Cette étude a permis d'autre part de révéler la problématique de la croissance en hauteur lente des plants en pépinière. Ce qui suscite l'intérêt de conduire des études pour évaluer l'effet des hormones de croissance les caractéristiques physiologiques et biologiques des plants de cette espèce en pépinière.

préoccupations a permis d'assurer l'arrosage et le suivi des plants depuis le stade de semis jusqu'à la fin de l'expérimentation. Qu'il trouve ici toute notre reconnaissance.

\section{CONFLIT DD'INTËTERET}

Cet article ne présente aucun conflit d'intérêt entre ses différents coauteurs

\section{REFFERENCES BIBLIOGRAPHIQUES}

Abdourhamane H, Dan Guimbo I, Morou B, Mahamane A, 20142. Potential germination and initial growth of Sclerocarya birrea (A. Rich.) Hochst, in Niger. Journal of Applied Biosciences 76:6433- 6443.

Alexandre OY, 1993. Quelques observations sur la physiologie des semences et des plantules forestières de la zone du Nazinon, pp. 203209. In : Les problèmes de semences forestières, notamment en Afrique. IUFRO Symposium, Ouagadougou, Burkina Faso, 2328 Nov. 1992, Backhuys Publishers, 471 p.

Assongba Y, Gjégo G.J., Sinsin B, (2013. Capacité de germination de Dialium guineense willd (Fabaceae) une espèce Agroforestière. Journal of Applied Biosciences 62: 4566 $4581.16 p$

Ahaton L E., Adjakpa JB, M'PO IM, Akpo EL, 2009. Effet des prétraitements des semences sur la germination de Prosopis africana (Guill., Perrot. et Rich.) Taub., (Césalpiniacées). TROPICULTURA, 2009, 27, 4, 233-238

Assogbadjo AE, Kyndt T, Sinsin V, Gheysen G, Van Damme $P, 2006$. Patterns of genetic and morphometric diversity in Baobab (Adansonia digitata) population across different climatic zones of Benin (West Africa), Ann. Bot 97 (5) 819-830.

Bastide B, Diallo BO. 1996. Essai comparatif de provenances de Faidherbia albdia Del., in : Peltier R. (Éd.), Les parcs Agroforestiers de Faidherbia albida, Cirad-Forêt, Baillarguet, France,

Billand $A$, Deframond $H$, 1990. Variabilité génétique d'Acacia albida (synonyme Faidherbia albida) en essais comparatifs de provenances au Burkina Faso, in : Physiologie des arbres et arbustes en zones arides et semi arides, Groupe d'étude de l'arbre, Paris, France, pp. 235-248

Bellefontaine R, Gamène C, Ouédraogo AS, Boffa JM, 1998. Vers une approche régionale des ressources génétiques forestières en Afrique Sub-Saharienne Pp. 155-160. Actes du premier atelier régional sur la conservation et l'utilisation durable des ressources génétiques forestières en Afrique de l'Ouest, Afrique Centrale et Madagascar. Centre National de Semences Forestières, Ouagadougou, Burkina Faso.

Bellefontaine R, 2005. Pour de nombreux ligneux, la reproduction sexuée n'est pas la seule voie : analyse de 875 cas - Texte introductif, tableau 
et bibliographie. Sécheresse Revue électronique, $\quad 3 E, \quad 60 \quad p$. http://www.secheresse.info/article.php3?id_arti cle $=2344$

Bationoa BA, Ouedraogoa JS., Guinko S, 2000. Longévité des graines et contraintes à la survie des plantules d'Afzelia africana Sm. dans une savane boisée du Burkina Faso. Ann. For. Sci. 58 (2001) 69-75

Bariteau M, 1992. Régénération naturelle de la forêt tropicale humide de Guyane : étude de la répartition spatiale de Qualearosa Aublet, Eperna falcata Aublet et Symphonia globulifera Linnaeus f. Ann. Sci. For. 49359 392.

Cavanaght T, 1987. Germination of hard-seeded (order Fabales). In : Germination of Australian native plant seed. Melbourne, Sydney, Australia, Inkata Press, p. 58-70.

Doucet JL, 2007. Le moabi, une espèce «phare» de l'exploitation forestière en Afrique centrale. Parcs et Réserves - Volume $62 \mathrm{n}^{\circ} 2$

Coppen J JW, and Hone, G A, 1995. Non-Wood Forest Products 2. Gum Naval Stores: Turpentine and Rosinfrom Pine Resin. Rome : FAO.

Dianda M, Ouédraogo SJ, Diop TA, 2010. Variation de la réponse à l'endomycorhization enpépinière des plants issus de deux semenciers de karité au Burkina Faso. International Journal of Biological and Chemical Sciences 4(5):17421752, http://ajol.info/index.php/ijbcs

Douma S, Chaibou.R, Mahamane A, Dibi HN, Mahamane S., 2010. Etat actuel de dégradation des peuplements de quatre espèces ligneuses fruitières en zone sahélosoudanienne du Niger: Reserve totale de Tamou. Rev. Ivoir.Sci. Tehno.16. 191-210

Diallo B, Joly HI., Mckey D, Hossaertmckey M, Chevallier MH, 2008. Variation des caractères biométriques des graines et des plantules de neuf provenances de Tamarindus indica $\mathrm{L}$. (Caesalpinioideae). Fruits, vol. 65 (3) 153

Diallo BO, 2001. Biologie de la reproduction et évaluation de la diversité génétique chez une légumineuse : Tamarindus indica $\mathrm{L}$. (Caesalpinioideae), Univ. Montpellier II, Sci. Tech. Languedoc, thèse, Montpellier, France, $119 p$.

El Hamdouni EM, Lamarti A, Badoc A, 2001. Germination in vitro du Fraisier (Fragaria $x$
Ananassa duch.) cvs 'chandler' et 'tudla' Bull. Soc. Pharm. Bordeaux, 140, 19-30

Dan Guimbo I., Ambouta K. J-M, Mahamane A., \& Larwanou M., 2011.Germination et croissance initiale de Neocarya macrophylla (Sabine) Prance, une espèce oléagineuse du Niger. Tropicultura, 2 (29) : 88-93.

FAO, 2006. Global Forest Ressources Assessment 2005. FAO, Rome, 320 p.

François W K, Nouhoun Z, Didier Z, Sawadogo L, 2019. Impact des activités anthropiques sur la diversité ligneuse et la structure de Vitellaria paradoxa Gaertn. C.F. dans le Chantier d'Aménagement Forestier de Cassou (Burkina Faso). Flora et Vegetatio Sudano-Sambesica 22, 3-15. DOI : 10.21248/fvss.22.62

Ganaba S, 2005. Impact des aménagements de conservation des eaux et des sols sur la régénération des ressources ligneuses en zone sahélienne et nord soudanienne du Burkina Faso. VertigO.DOI :10.4000/vertigo.431

Garba A, Abdou A, Abdou L, Mahamane A, 2019. Perceptions et usages socioéconomiques du tamarinier (Tamarindus indica L.) dans le SudOuest du Niger : Implications pour une domestication et une conservation durable. Journal of Animal \& Plant Sciences (J.Anim.Plant Sci.), 40(2) : 6584-6602

Karim S, Mahamane A, Morou M, Saadou M, 2010. Dynamique de l'occupation des terres et caractéristiques de la végétation dans la Commune rurale de Simiri (région de Tillabery, Niger. Annales de l'Université Abdou Moumouni, Tome XI-A : 166-177.

Getahun A., 1990. The role of wild plants in the native diet in Ethiopia. Agroecosystem, 1: 45-56.

Koffi CK, Djoudi H, Gautier D, 2016. Landscape diversity and associated coping strategies during food shortage periods: evidence from the Sudano-Sahelian region of Burkina Faso. Reg Environ Change 16:1-12.

Kafando WC.BI, 2016. Etude des caractéristiques morphologiques des graines et des paramètres de croissance de Prosopis a/ricana (Guill. \& Perr.) Taub. en pépinière de vingt provenances de trois pays sahéliens Ouest Africains. Diplôme d'Ingénieur du Développement Rural : Option Eaux et Forêts.

Lykke A M, Kristensen M K, Ganaba S, 2004. Valuation of the local dynamics of 56 woody species in 
the Sahel. Biodiversity and Conservation, 13: 1961-1990

Lopez M, Humara JM, Casares A, Majada J, 2000. The effect of temperature and water stress on laboratory germination of Eucalpytus globulus Labill. Seeds of different sizes, Ann. For. Sci. 57245-250.

Saadou M, 1990. La végétation des milieux drainés nigériens à l'est du fleuve Niger. Thèse de doctorat, Université Niamey, Niger, 393 p.

Millogo D, 2014. Etudes des caractéristiques morphologiques et de la viabilité des semences de Parkia biglobosa (Jacq.) R. Br. ex G. Don. germoplasme de conservation à long terme $A 4^{\circ} \mathrm{C}$. Mémoire de fin d'étude, IDR/IJPB.42p.

Ouédraogo A, Thiombiano A, Hahn-hadjali Guinko S, 2006. Régénération sexuée de Boswellia dalzielii Hutch., un arbre médicinal de grande valeur au Burkina-Faso. Bois et Forêts des Tropiques, 289, 3, 41-52.

Orozco-Segovia A, Vazquez-Yanes C, 1993. Effect of moisture on longevity in seeds of some rain forest species, Biotropica 22 215-216.

Pierre R, 2003. Physiologie et architecture des racines. In Actes du 9è congrès de l'APEVC, Igualada, Espagne, 22-29.

Tran VN, Cavanagh AK, 1984. Structural aspects of dormancy: In: D.R. Murray (ed.) Seed Physiology. V. II. Academic Press, Melbourne, pp.1-44.

Todd-Bockarie AH, Duryea ML, 1993. Seed pretreatment methods to improve germination of the multipurpose West African forest species Dialium guineense. For. Ecolo. Manage. 57 (1-4), 257-273.

Vetaas OR, 1992. Microsites effects of tree and shrubs in dry savannas, J. Végét. Sci. 3 337-344

World Bank, 2003 World development report. Sustainable development in a dynamic world : transforming institutions, growth, and quality of life. New Delhi, Oxford University Press.

Werker E, 1981. Seed dormancy as explained by the anatomy of embryo envelopes. Isr. J. Bot., 29: 22-44. 